\title{
Proteinuria as a Marker of Chronic Kidney Disease: A Single Centre Single Day Screening Experience for 9 Years in Varanasi
}

\author{
Rai Pradeep K ${ }^{1 *}$, Rai Punam² and Bedi Sonam ${ }^{3}$ \\ ${ }^{1}$ Senior Consultant Nephrologist, Department of Nephrology, Opal Hospital, Varanasi, \\ India \\ ${ }^{2}$ Senior Clinical Physiologist \& Infertility expert, Opal Hospital, Varanasi, India \\ ${ }^{3}$ Senior Data Analyst, Department of Medicine, Institute of Medical Sciences, Banaras \\ Hindu University, Varanasi, India
}

\section{Research Article \\ Volume 3 Issue 4}

Received Date: November 27, 2018

Published Date: December 21, 2018 DOI: 10.23880 /oajun-16000152

*Corresponding author: Pradeep Kumar Rai, M.B.B.S, MD, DM, D.N.B, F.A.S.N, Senior Consultant Nephrologist, Department of Nephrology, Opal Hospital, Kakarmatta, Varanasi- 221004, India, Tel: +91-9336913486, Email: pradnephro@gmail.com; pradeepk.rai@gmail.com

\section{Abstract}

Introduction: Chronic kidney disease is an asymptomatic devastating medical illness which often results in delayed recognition with direct social and economic consequences for the patients, their families and the country. Proteinuria is one of the most prognostically significant biomarkers of kidney disease outcomes and even cardiovascular disease and death. The present study is a hospital camp-based screening of general population for the detection of proteinuria for 9 years (2008-11 and 2014-18).

Methods: The study constituted a total of 1081 healthy adults of Varanasi. Information was recorded on sociodemographic and clinical profile of subjects. Prevalence of proteinuria was found. Further, significant predictors of proteinuria were estimated using binary logistic regression analysis.

Results: There was almost equal proportion (50\% each) of both the sexes in the study cohort with median age of 40 years. Proteinuria was present in $29.2 \%$ subjects. Male gender, smoking status, diabetes and abnormal creatinine levels came out as significant predictors of proteinuria.

Discussion: Evidences have recommended proteinuria as a surrogate for kidney disease progression. Using the advantage of World Kidney Day, we collated screening data of 9 years and found high prevalence of proteinuria and its significant interlinked predictors like diabetes, hypertension, smoking which are all prelude to CKD and several other chronic outcomes. Such potentially modifiable risk factors are easily detectable in screening programs and can be mitigated, which is a pre requisite to prevent future health adversities.

Keywords: Proteinuria; Serum Creatinine; Diabetes Mellitus; Smoking; Hypertension; Chronic Kidney Disease 
Abbreviations: CKD: Chronic Kidney Disease; DOQI: Dialysis Outcomes Quality Initiative; NKF: National Kidney Foundation; AKI: Acute Kidney Injury; DM: Diabetes Mellitus; HTN: Hypertension; RBS: Random Blood Sugar; BP: Blood Pressures; BMI: Body Mass Index; SPSS: Statistical Package for Social Sciences; IQR: Inter Quartile Range; OR: Odds Ratio; GFR: Glomerular Filtration Rate ESRD: End-Stage Renal Disease.

\section{Introduction}

Chronic kidney disease (CKD) is a devastating medical illness with direct social and economic consequences for the patients, their families and the country due to its associated adverse clinical outcomes, poor quality of life and high healthcare costs $[1,2]$.

Proteinuria is now a central part of screening for and monitoring of kidney disease and is one of the most prognostically significant biomarkers of kidney disease outcomes and even cardiovascular disease and death $[3,4]$. Studies suggest populations at increased risk for CKD (i.e., those with diabetes mellitus, hypertension, or family history of CKD) should be screened for micro albuminuria, at least annually, as a part of their regular health examination [5].

CKD is asymptomatic in the early stages, which often results in delayed recognition and further treatment hence, early detection of kidney disease via screening programs is widely recommended [6,7]. The first set of clinical practice guidelines developed under National Kidney Foundation (NKF) to expand its Dialysis Outcomes Quality Initiative (DOQI) K/DOQI recommended that all individuals should be assessed, as part of routine health encounters, to determine whether they are at increased risk for developing kidney disease, based on sociodemographic factors and also individuals identified as being "at increased risk should undergo testing for markers of kidney damage," specifically for proteinuria for the probable detection of CKD [8].

The lack of community-based screening programs for CKD, results in patient detection at advanced and irreversible stages of CKD and ESRD $[9,10]$. We conducted a hospital camp-based screening of general population on World Kidney Day for nine years (2008-11 and 2014-18) for the detection of proteinuria.

\section{Methods}

The screening camp was conducted at Opal hospital in Varanasi as a part of World Kidney Day celebration based on its theme every year. The study subjects constituted apparently healthy subjects of age $\geq 18$ years. The information about the screening camp was spread via electronic media and newspapers to improve the awareness of the populace about the need for routine screening for proteinuria and hence, CKD due to its asymptomatic nature.

Approval to conduct the screening camp was obtained from the Ethical and Research Committee of Opal Hospital. Consecutively, the participants who presented themselves for screening were enrolled in the exercise. All the participants provided informed consent. Subjects with symptomatic acute kidney injury (AKI) or CKD or transplanted kidneys were excluded from the screening. A structured questionnaire was used to obtain information on age, sex, height, weight, smoking status, history of diabetes mellitus (DM) and hypertension (HTN), familial history of kidney disease. Clinical investigations like, random blood sugar (RBS), urinalysis and serum creatinine levels were performed. Systolic and diastolic blood pressures (BP) were measured on the day of screening. Significant differences in the mean/median levels of continuous variables were checked on the basis of proteinuric and non-proteinuric groups. These variables were further categorized for better clinical interpretation and usage in the regression models.

Age was divided into 2 categories (in complete years), e.g. $<45$ and $\geq 45$. Body mass index (BMI) was calculated using height and weight and divided based on presence of obesity into 2 categories (in $\mathrm{kg} / \mathrm{m} 2$ ): non-obese ( $\leq 29.99$ ) and obese (>29.99). Diabetes was defined as the use of glucose lowering medicine and/or RBS $\geq 200$. HTN was defined as the use of any hypertensive and/or based on systolic (SBP) and diastolic BP (DBP) and further, it was divided into 3 categories as normal, elevated BP and hypertension [11]. Smoking status, DM and family history of kidney disease was dichotomized as: yes (present) and no (absent). Two milliliters of blood sample was collected from each participant through venipuncture to investigate for serum creatinine level. Serum creatinine level $(\mathrm{mg} / \mathrm{dl})$ was divided in 2 groups: normal $(<1.30)$ and abnormal $(\geq 1.30)$.

A urine dipstick (Medi-Test Combi 9-Macherey Nagel, Duren, Germany) was performed for each individual. The outcome variable of interest was presence of proteinuria as it is an important biomarker for the occurrence of CKD. Proteinuria was regarded as significant if $1+$ and above and categorized as present or absent [12]. Participants with elevated BP, HTN, DM and proteinuria were referred 
to a renal physician or nephrologist of their choice for further evaluation and treatment.

\section{Statistical Analyses}

All the statistical analysis was performed on Statistical Package for Social Sciences (SPSS) version 22 (SPSS Inc., Chicago, IL, USA). Normality of the continuous variables was checked using Kolmogorov Smirnov test. Continuous non-parametric variables were displayed as median (inter quartile range (IQR)) and categorical variables in percentages. Mann Whitney $U$ test was used to assess differences between medians. Association between categorical variables with proteinuria was checked using Pearson's chi square test and Fisher's exact correction was applied as appropriate. All p values less than 0.05 were considered statistically significant. To determine the contribution of various variables associated with the presence of outcome (proteinuria) was seen using bivariable binary logistic regression analysis. The predictors significant in bi-variable analysis (at 10\%) were taken into the final multi-variable binary logistic regression analysis. 95\% confidence intervals of the estimated odds ratio (OR) were also obtained along with the corresponding $\mathrm{p}$-values.

\section{Results}

A total of 1081 participants were included in the screening exercise with 565 males (52.3\%) and 516 females $(47.7 \%)$. The prevalence of proteinuria in the cohort was found to be $29.2 \%$. Table 1 showed the sociodemographic and clinical profile (of continuous variables) of the study subjects and their significant differences, checked on the basis of proteinuria.

\begin{tabular}{|c|c|c|c|c|}
\hline \multirow{2}{*}{$\begin{array}{c}\text { Characteristics } \\
\text { (Median (IQR)) }\end{array}$} & \multicolumn{2}{|c|}{ Proteinuria } & \multirow{2}{*}{ Total } & \multirow{2}{*}{ Mann Whitney p value } \\
\cline { 2 - 3 } & Yes & No & $40(30-52)$ & 0.001 \\
\hline Age (years) & $44(35-55)$ & $40(30-50)$ & $107(95-128)$ & $<0.001$ \\
\hline BMI (Kg/m2) & $21.09(17.53-25.94)$ & $23.31(18.95-27.62)$ & $22.71(18.50-27.39)$ & $<0.001$ \\
\hline RBS & $115(99-179)$ & $106(94-120)$ & $120(110-140)$ & $<0.001$ \\
\hline SBP & $130(120-140)$ & $120(110-130)$ & $80(70-90)$ & $<0.001$ \\
\hline DBP & $80(70-90)$ & $80(70-90)$ & $0.90(0.70-1.10)$ & 0.035 \\
\hline Creatinine (mg/dl) & $0.80(0.60-1.10)$ & $0.90(0.70-1.10)$ & 0 \\
\hline
\end{tabular}

Table 1: Descriptive statistics of continuous variables according to proteinuria outcome.

Median (IQR) age of the participants was 40 (30-52) years with significantly higher age in proteinuric group than non-proteinuric group ( $\mathrm{p}=0.001)$. There were $58 \%$ subjects $<45$ years of which nearly quarter $(25.4 \%)$ subjects were proteinuric. Age was statistically associated with presence of proteinuria (Table 2). There were nearly equal proportion of both sexes in the study sample but proteinuria was found more prominent in males than females (35.9\% vs. $21.9 \%, \mathrm{p}<0.001)$. Median (IQR) BMI was $22.71(18.50-27.39) \mathrm{kg} / \mathrm{m}^{2}$ with higher level in non protenuric group $(\mathrm{p}<0.001)$. The prevalence of obesity in the entire study cohort was $15.4 \%$. Obesity was not associated with proteinuria occurrence $(\mathrm{p}=0.629)$.

\begin{tabular}{|c|c|c|c|c|}
\hline Characteristics & $\mathbf{N}$ & \% distribution & \% CKD & Pearson's $\chi^{2} p$ value \\
\hline \multicolumn{5}{|l|}{ Age (years) } \\
\hline$<45$ & 627 & $58.00 \%$ & $25.40 \%$ & \multirow{2}{*}{0.001} \\
\hline$\geq 45$ & 454 & $42.00 \%$ & $34.60 \%$ & \\
\hline \multicolumn{5}{|l|}{ Gender } \\
\hline Male & 565 & $52.30 \%$ & $35.90 \%$ & \multirow{2}{*}{$<0.001$} \\
\hline Female & 516 & $47.70 \%$ & $21.90 \%$ & \\
\hline \multicolumn{5}{|l|}{ BMI } \\
\hline Obese & 157 & $15.40 \%$ & $28.70 \%$ & \multirow{2}{*}{0.629} \\
\hline Non-Obese & 863 & $84.60 \%$ & $30.60 \%$ & \\
\hline \multicolumn{5}{|l|}{ Smoking } \\
\hline Yes & 59 & $5.70 \%$ & $59.30 \%$ & \multirow{2}{*}{$<0.001$} \\
\hline No & 982 & $94.30 \%$ & $27.80 \%$ & \\
\hline
\end{tabular}

Rai Pradeep K, et al. Proteinuria as a Marker of Chronic Kidney Disease: A Single Centre Single Day Screening Experience for 9 Years in Varanasi. J Urol Nephrol 2018, 3(4): 000152. 


\begin{tabular}{|c|c|c|c|c|}
\hline Yes & 40 & $3.80 \%$ & $52.50 \%$ & \multirow{2}{*}{0.001} \\
\hline No & 1010 & $96.20 \%$ & $28.80 \%$ & \\
\hline \multicolumn{5}{|l|}{ DM } \\
\hline Yes & 136 & $12.60 \%$ & $62.50 \%$ & \multirow{2}{*}{$<0.001$} \\
\hline No & 941 & $87.40 \%$ & $24.50 \%$ & \\
\hline \multicolumn{5}{|l|}{ HTN } \\
\hline Yes & 176 & $17.20 \%$ & $39.80 \%$ & \multirow{2}{*}{0.002} \\
\hline No & 847 & $82.80 \%$ & $28.20 \%$ & \\
\hline \multicolumn{5}{|c|}{ Serum creatinine (mg/dl) } \\
\hline$<1.30$ & 966 & $89.40 \%$ & $27.20 \%$ & \multirow{2}{*}{$<0.001$} \\
\hline$\geq 1.30$ & 115 & $10.60 \%$ & $46.10 \%$ & \\
\hline
\end{tabular}

Table 2: Association of proteinuria with various co-factors.

Overall SBP and DBP levels were recorded normal in the screening but there was a significant difference in the levels based on presence of proteinuria $(\mathrm{p}<0.001$ for each). $7.6 \%$ subjects were having elevated BP whereas $69.8 \%$ were hypertensive; of these $38.5 \%$ and $32.2 \%$ were having proteinuria $(\mathrm{p}=0.002)$ respectively. Median RBS level was 107 (95-128) mg/dl with higher side in the proteinuria group $(\mathrm{p}<0.001)$. A total of $12.6 \%$ subjects were identified with DM. More than 3/5th diabetics were suffering with proteinuria $(\mathrm{p}<0.001)$.

The study sample consisted of 5.7\% smokers. Further, smoking was highly associated with occurrence of proteinuria compared to non-smoking $(59.3 \%$ vs. $27.8 \%$, $\mathrm{p}<0.001$ ). Family history of kidney disease was found in only $3.8 \%$ subjects with more than half $(52.5 \%)$ having proteinuria $(\mathrm{p}=0.001)$. Median creatinine levels were $0.90(0.70-1.10)$ with significantly lower values in proteinuria group $(\mathrm{p}=0.035)$. Nearly, one-tenth $(10.6 \%)$ subjects were having abnormal creatinine levels $(\geq 1.30)$ and out of these, more than 2/5th (46.1\%) were proteinuric $(\mathrm{p}<0.001)$. Prevalence of proteinuria in the different characteristics of study cohort is shown in bar chart (Figure 1).

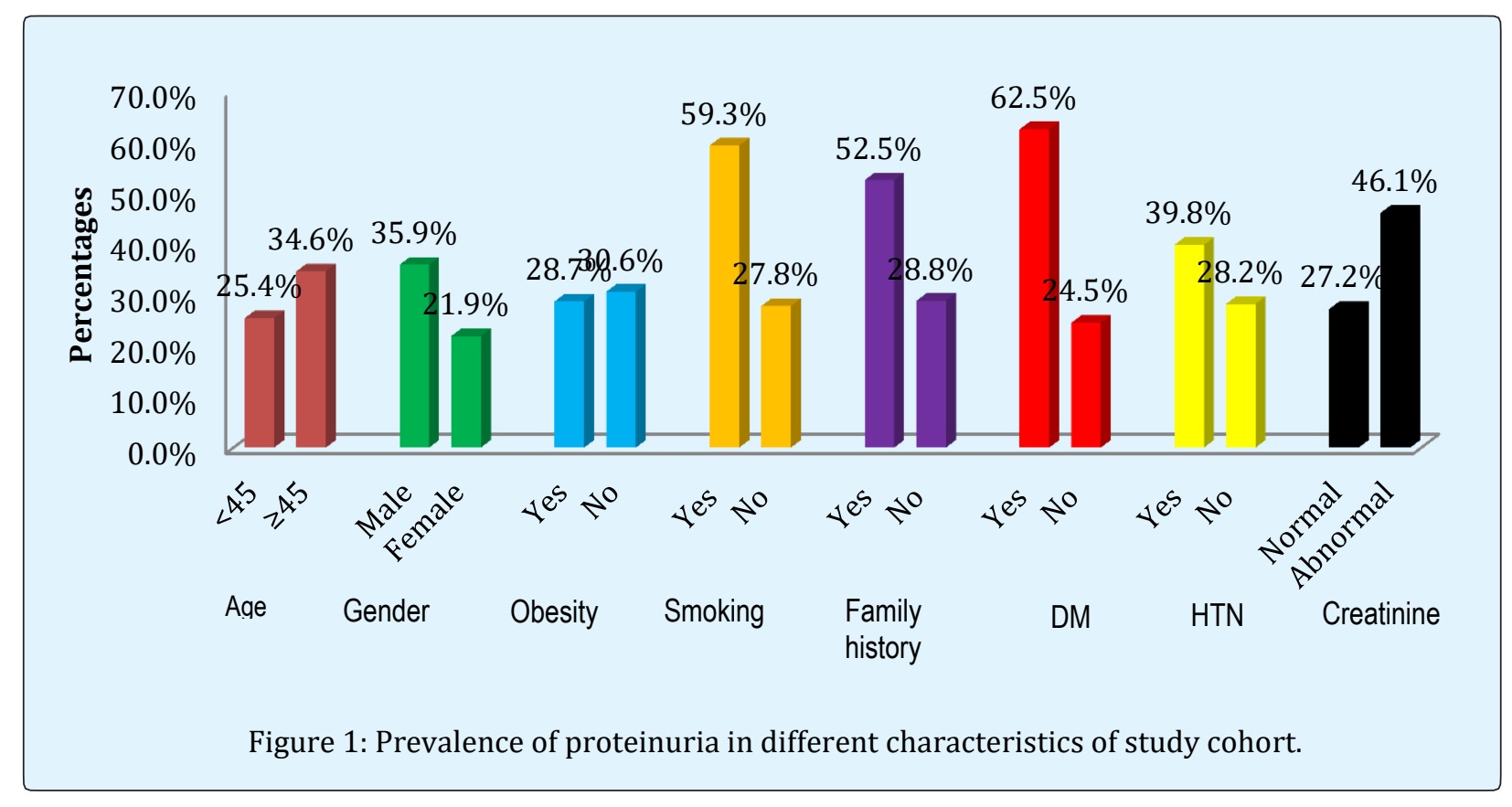

All the eight factors were considered in the bi-variable analysis (Table 3). Age, sex, DM, HTN, smoking, family history of kidney disease, creatinine level came as individual risk factors for the occurrence of proteinuria. Further these were included in the multi-variable analysis. In the multi-variable model (Table 3), we found 
males were more prone to suffer from proteinuria compared to females (OR: 1.677, CI: 1.243-2.262). Smokers and diabetics were associated with a 3 -fold and 4 -fold increase in the odds of having proteinuria respectively (OR: 3.417 , CI: 1.937-6.027 and OR: 4.777, CI:
3.210-7.111). Subjects with abnormal creatinine levels $(\geq 1.30)$ were associated with doubling the odds of having proteinuria (OR: 2.002, 95\% CI: 1.295-3.094) compared to normal creatinine levels.

\begin{tabular}{|c|c|c|}
\hline \multirow{2}{*}{ Characteristics } & \multicolumn{2}{|c|}{ P-values OR (95\% CI of OR) } \\
\hline & Bi-variable predictors & Multi-variable predictors \\
\hline \multicolumn{3}{|l|}{ Age in years (Ref: $<45$ ) } \\
\hline \multirow{2}{*}{$\geq 45$} & 0.001 & \multirow{2}{*}{$*$} \\
\hline & $1.556(1.195-2.026)$ & \\
\hline \multicolumn{3}{|l|}{ Gender (Ref: Female) } \\
\hline \multirow{2}{*}{ Male } & 0 & 0.001 \\
\hline & $2.000(1.526-2.621)$ & $1.677(1.243-2.262)$ \\
\hline \multicolumn{3}{|l|}{ BMI (Ref: Non obese) } \\
\hline \multirow{2}{*}{ Obese } & 0.629 & \multirow{2}{*}{$*$} \\
\hline & $.912(.627-1.326)$ & \\
\hline \multicolumn{3}{|l|}{ DM (Ref: No) } \\
\hline \multirow{2}{*}{ Yes } & 0 & 0 \\
\hline & $5.123(3.512-7.473)$ & $4.777(3.210-7.111)$ \\
\hline \multicolumn{3}{|l|}{ HTN (Ref: No) } \\
\hline \multirow{2}{*}{ Yes } & 0.003 & \multirow{2}{*}{$*$} \\
\hline & $1.680(1.199-2.353)$ & \\
\hline \multicolumn{3}{|l|}{ Smoking (Ref: No) } \\
\hline \multirow{2}{*}{ Yes } & 0 & 0 \\
\hline & $3.787(2.212-6.485)$ & 3.417 (1.937-6.027) \\
\hline \multicolumn{3}{|c|}{ Family history of renal disease (Ref: No) } \\
\hline \multirow{2}{*}{ Yes } & 0.002 & \multirow{2}{*}{$*$} \\
\hline & $2.731(1.447-5.155)$ & \\
\hline \multicolumn{3}{|l|}{ Creatinine (Ref: <1.30) } \\
\hline \multirow{2}{*}{$\geq 1.30$} & 0 & 0.002 \\
\hline & $2.285(1.542-3.385)$ & $2.002(1.295-3.094)$ \\
\hline
\end{tabular}

Table 3: Bi-variable and multi-variable predictors for the occurrence of proteinuria.

\section{Discussion}

Chronic Kidney Disease is an enormous public health issue typically evolving over many years, with a long latent period when the disease is clinically silent and therefore diagnosis, evaluation and treatment is based mainly on biomarkers that assess kidney function. Glomerular filtration rate (GFR) remains the ideal marker of kidney function. Unfortunately measuring GFR is time consuming and not easily measurable in most clinical or research settings, other biomarkers such as proteinuria may precede kidney function decline and has been demonstrated to have strong associations with disease progression and outcomes [13]. The aim of the present study was to find the prevalence of proteinuria in apparently healthy population of Varanasi. The study participants willingly participated in hospital based screening camp on a single day from 2008-11 and 201418. Further, we found the factors associated with occurrence of proteinuria which is a prelude to kidney disease progression, cardiovascular disease and other interlinked chronic diseases.

Our study reported overall a very high prevalence of proteinuria as $29.2 \%$ which is an over estimate compared to other published studies although our previous similar studies reported even higher prevalence (i.e. higher than $30 \%)$, though similar North Indian studies from different authors reported quite low prevalence of proteinuria [14$16]$. 
Diabetes and hypertension are the key risk factors of CKD, cardio-vascular diseases and other chronic disease worldwide in all high-income and middle-income countries, and also in many low-income countries [17]. Unsurprisingly our data also showed that diabetics and hypertensive were more prone to be proteinuric in bivariable analysis though only DM was retained in the multi-variable model holding a very high risk (4-fold) for proteinuria occurrence. DM and HTN can be known easily through blood pressure and blood glucose measurement. Early detection of these factors can allow prompt initiation of appropriate educational and medical therapies, which may, in turn, impact positively on long term kidney and other adverse outcomes.

Smoking being a well-known risk factor for many chronic diseases is also proven to play an important role in renal diseases. There is growing evidence that smoking increases the risk of proteinuria along with renal functional deterioration [18]. Present analysis added on similar evidences, with smokers around thrice more likely to suffer with proteinuria compared to non-smokers.

The study sample comprised of subjects between ages $18-90$ years with $42 \%$ subjects aging $\geq 45$ years hence vulnerable to the age related glomerulosclerosis and decline in kidney function. Nearly one-third (34.6\%) aged above 45 years were found to be suffering with proteinuria $(\mathrm{p}=0.001)$ with about $56 \%$ increase in the odds of developing proteinuria in the bi-variable analysis. Clearly, aging being a strong non-modifiable risk factor for proteinuria but came significant only in bi-variable analysis and not multi-variable analysis (Table 3). Further work is required to determine whether renal impairment in elderly subjects is associated with or causes other conditions.

Serum creatinine being a convenient index of kidney function [19] was found associated with doubling the risk of occurrence of proteinuria (Table 3) which is in consensus with published literature [20]. 10.6\% subjects had abnormal serum creatinine levels, of which $46.1 \%$ were identified with proteinuria (Table 2). Henceforth, subjects with even minimal creatinine elevation are suggested for frequent health checkups as it may indicate presence of clinically significant kidney disease, which could be prevented from further progression to higher stages of CKD.

Men are more prone to develop renal diseases and to progress to end-stage renal disease (ESRD) than women, when all-cause incidence rates are considered [21,22]. Our analysis revealed nearly 1.7 times higher risk of proteinuria in males compared to females. Similar outnumbering of proteinuria in males than females has been shown earlier.

Subjects with family history of kidney disease were twice more likely to suffer from proteinuria in the bivariable analysis but the result was non-significant in multi-variable analysis (Table 3). Family history of kidney disease is an important but non modifiable risk factor for both proteinuria and CKD. It is suggested that subjects with familial kidney disease history should go for frequent monitoring to detect presence of any asymptomatic disease.

The magnitude of existing burden of ESRD, the projections for increasing incidence of CKD, the high cost and lack of resources for renal replacement therapy in most low- and middle-income countries, call for the prevention of CKD and its risk factors using an approach based on awareness, early detection and effective treatment [23]. Evidences have recommended proteinuria as a surrogate for kidney disease progression although further research is needed to define additional contexts in which changes in proteinuria can be expected to predict treatment effect [24]. Using the advantage of World Kidney Day, which is intended to raise awareness and increase the detection of CKD and its associated predictors, we collated screening data of 9 years considering proteinuria as an event of interest and found the prevalence of proteinuria along with its significant interlinked predictors like diabetes, hypertension which are all prelude to CKD and several other chronic outcomes. The objective was to identify what risk factors, present in the community, are associated with the development of proteinuria and thereby CKD which are potentially modifiable, in order to intervene at the earliest possible stage and prevent future health impairment.

\section{Limitations}

The data collection was done in a cross sectional way on a single day measure every year for nine years. Single day measurements of serum creatinine and urinary markers of kidney damage are likely to overestimate the true values.

\section{Acknowledgements}

We would like to thank medical officers and staff of Opal hospital: Dr Shashi Kumar Gupta, Dr Hemant Kumar Rai, Dr Zafar Raja, Dr Santosh Mishra, Dr Surendra Nath Gupta, Mrs. Arti Maurya and Mr. Bansi Dhar Pandey who 
have helped with evaluation of subjects in the screening camp.

\section{References}

1. Schieppati A, Remuzzi G (2005) Chronic Renal Diseases as a Public Health Problem: Epidemiology, Social, and Economic Implications. Kidney Int Suppl 98: 7-10.

2. Sonam B, Pandey CM, Gupta A, Prasad N, Kaul A, et al. (2015) Renal Transplant: Tacrolimus Use and Two Weeks Post-Transplant Serum Creatinine Levels Predict Early Acute Rejections. Indian Journal of Transplantation 9(3): 90-94.

3. Baumgarten $M$, Gehr $T$ (2011) Chronic Kidney Disease: Detection and Evaluation. Am Fam physician 84(10): 1138-1148.

4. Ferguson MA, Waikar SS (2012) Established and Emerging Markers of Kidney Function. Clin Chem 58(4): 680-689.

5. Eknoyan G, Hostetter T, Bakris GL, Hebert L, Levey AS, et al. (2003) Proteinuria and Other Markers of Chronic Kidney Disease: A Position Statement of the National Kidney Foundation (NKF) and the National Institute of Diabetes and Digestive and Kidney Diseases (NIDDK) 1. American Journal of Kidney Diseases 42(4): 617-622.

6. Rai PK, Jindal PK, Rai P, Pramod Kumar Rai, Rai SN (2014) Screening of Chronic Kidney Disease (CKD) in General Population on World Kidney Day on Three Consecutive Years: A Single Day Data. International Journal of Medicine and Public Health 4(2): 167-170.

7. Mendelssohn DC (2005) Coping with the CKD Epidemic: The Promise of Multidisciplinary TeamBased Care. Nephrology Dialysis Transplantation 20(1): 10-12.

8. Levey, Andrew S et al. 2002. K/DOQI Clinical Practice Guidelines for Chronic Kidney Disease: Evaluation, Classification, and Stratification. American Journal of Kidney Diseases 39(2).

9. Modi GK, Jha V (2006) The Incidence of End-Stage Renal Disease in India: A Population-Based Study. Kidney Int 70(12): 2131-2133.

10. Rai PK, Rai P, Bedi S (2018) Prevalence and Risk Factors of Chronic Kidney Disease: A Single Day Screening on World Kidney Day for Four Consecutive
Years in Varanasi. Urology \& Nephrology Open Access Journal 6(6): 167-171.

11. Paul KW, Carey RM, Aronow WS, Casey DE, Collins KJ, $\begin{array}{lll}\text { et } & \text { al. } & \text { (2018) }\end{array}$ ACC/AHA/AAPA/ABC/ACPM/AGS/APhA/ASH/ASPC /NMA/PCNA Guideline for the Prevention, Detection, Evaluation, and Management of High Blood Pressure in Adults: A Report of the American College of Cardiology/American Heart Association Task Force on Clinical Practice Guidelines. Journal of the American College of Cardiology 71(19): 127-248.

12. Ulasi II, Ijoma CK, Onodugo OD, Arodiwe EB, Ifebunandu NA, et al. (2013) Towards Prevention of Chronic Kidney Disease in Nigeria: A CommunityBased Study in Southeast Nigeria. Kidney International Supplements 3(2): 195-201.

13. Lopez-Giacoman S, Madero M (2015) Biomarkers in Chronic Kidney Disease, from Kidney Function to Kidney Damage. World J Nephrol 4(1): 57-73.

14. Ayodele OE, Okunola 00, Afolabi MO, Oluyombo R, Gbadegesin BA, et al. (2011) Prevalence of Hypertension, Diabetes and Chronic Kidney Disease in Participants of the 2009 World Kidney Day Screening Exercise in Southwest Nigeria. Hong Kong Journal of Nephrology 13(2): 55-63.

15. Singh AK, Farag YM, Mittal BV, Subramanian KK, Reddy SR, et al. (2013) Epidemiology and Risk Factors of Chronic Kidney Disease in India-results from the SEEK (Screening and Early Evaluation of Kidney Disease) Study. BMC Nephrol 14: 114.

16. Singh NP, Ingle GK, Saini VK, Jami A, Beniwal P, et al. (2009) Prevalence of Low Glomerular Filtration Rate, Proteinuria and Associated Risk Factors in North India Using Cockcroft-Gault and Modification of Diet in Renal Disease Equation: An Observational, CrossSectional Study. BMC Nephrol 10(1): 4.

17. Webster AC, Nagler EV, Morton RL, Masson P (2017) Chronic Kidney Disease. The Lancet 389(10075): 1238-1252.

18. Orth SR (2002) Smoking and the Kidney. Journal of the American Society of Nephrology 13(6): 16631672.

19. Abcar AC, Chan L, Yeoh H (2004) What To Do for the Patient with Minimally Elevated Creatinine Level?. Perm J 8(1): 51-53. 
20. Lin CC, Li CI, Liu CS, Lin WY, Lin CH et al. (2017) Development and Validation of a Risk Prediction Model for End-Stage Renal Disease in Patients with Type 2 Diabetes. Scientific Reports 7(1): 10177.

21. Iliescu R, Reckelhoff JF (2008) Sex and the Kidney. Hypertension 51(4): 1000-1001.

22. Pounds LL, Teodorescu VJ (2013) Chronic Kidney Disease and Dialysis Access in Women. J Vasc Surg 57(4): 49-53.
23. Ležaić $V$, Bajčetić $S$, Peruničić-Peković G, Bukvić D, Dimković N, et al. (2012) Screening of Elderly for Chronic Kidney Disease. Kidney and Blood Press Res 35(6): 497-503.

24. Levey AS, Cattran D, Friedman A, Miller WG, Sedor J, et al. (2009) Proteinuria as a Surrogate Outcome in CKD: Report of a Scientific Workshop Sponsored by the National Kidney Foundation and the US Food and Drug Administration. Am J Kidney Dis 54(2): 205226. 